\title{
Radiative electron capture in the first-forbidden unique decay of ${ }^{81} \mathrm{Kr}$
}

\author{
S. Mianowski, ${ }^{1}$ E. Werner-Malento,,${ }^{1}{ }^{*}$ A. Korgul,${ }^{1}$ M. Pomorski, ${ }^{1}$ K. Pachucki, ${ }^{1}$ M. Pfützner,${ }^{1, \dagger}$ B. Szweryn, ${ }^{1}$ J. Żylicz, ${ }^{1}$ \\ P. Hornsh $\varnothing \mathrm{j},{ }^{2}$ T. Nilsson, ${ }^{3,4}$ and K. Rykaczewski ${ }^{5}$ \\ ${ }^{1}$ Faculty of Physics, University of Warsaw, Hoza 69, 00-681 Warsaw, Poland \\ ${ }^{2}$ Institute of Physics and Astronomy, University of Aarhus, DK-8000 Aarhus C, Denmark \\ ${ }^{3}$ Fundamental Physics, Chalmers University of Technology, Gothenburg, Sweden \\ ${ }^{4}$ CERN, EP-Division, CH-1211, Geneva 23, Switzerland \\ ${ }^{5}$ Physics Division, Oak Ridge National Laboratory, Oak Ridge, Tennessee 37831, USA
}

(Received 9 August 2010; published 12 October 2010)

\begin{abstract}
The photon spectrum accompanying the orbital $K$-electron capture in the first-forbidden unique decay of ${ }^{81} \mathrm{Kr}$ was measured. The total radiation intensity for photon energies larger than $50 \mathrm{keV}$ was found to be $1.42(22) \times 10^{-4}$ per $K$ capture. Both the shape of the spectrum and its intensity relative to the ordinary, nonradiative capture rate are compared to theoretical predictions. The best agreement is found for the recently developed model that employs the length gauge for the electromagnetic field.
\end{abstract}

DOI: 10.1103/PhysRevC.82.044308

PACS number(s): 23.20.Nx, 23.40.-s, 27.50.+e, 29.30.Kv

\section{INTRODUCTION}

Radiative electron capture (REC) is a process in which one of the orbital electrons is captured by the atomic nucleus and in addition to an electron neutrino a photon is emitted [1]. Because of the three-body character of the process, the energy spectrum of these photons is continuous, reaching up to the maximum value of the decay energy $Q_{\mathrm{EC}}$ reduced by the binding energy of the captured electron in the daughter atom, $B(n l)$. Such a radiative process occurs with a probability of the order of $10^{-4}$ with respect to the ordinary, radiationless electron capture.

Early studies have shown, for numerous decays involving allowed nuclear transitions (nuclear spin changes by $\Delta J=0$, 1 with no parity change), that the intensity and shape of the REC spectrum can be well understood by assuming that radiation is emitted by a captured electron [internal bremsstrahlung (IB)] [1]. However, a measurement of REC in the case of the forbidden nuclear transition in ${ }^{41} \mathrm{Ca}$ [2] revealed a strong disagreement with existing models. Moreover, the most advanced theoretical model of IB, developed by Zon and Rapoport for an arbitrary degree of forbiddenness [3,4], showed the largest deviation from the experiment. The EC decay of ${ }^{41} \mathrm{Ca}$ belongs to a category of first-forbidden unique (1u) transitions $\left(\Delta J=2, \pi_{i} \pi_{f}=-1\right)$. Unique transitions are of special interest, because in the probability ratio of radiative to nonradiative decays, the nuclear matrix elements cancel out and the process should be governed solely by electromagnetic interactions. For ${ }^{41} \mathrm{Ca}$, the total probability of the REC process, per nonradiative decay, was found to be six times larger than the detailed prediction of the Zon and Rapoport model [2].

To explain this finding, a hypothesis of the so-called detour transitions was considered by Kalinowski and co-workers [5,6] following an idea of Ref. [7]. According to it, a large part of

\footnotetext{
*Present address: Institute of Physics, Polish Academy of Science, Al. Lotników 32/46, 02-668 Warsaw, Poland.

†pfutzner@fuw.edu.pl
}

the radiation is emitted by the nucleus in addition to the IB mechanism. It was argued that such a nuclear contribution is particularly significant in the case of $1 u$ decays, where the combination of an allowed $G T$ EC decay with a nuclear $E 1$ $\gamma$ transition, may compete with the direct nuclear $1 u$ transition accompanied by an IB photon. Apparently, for the case of ${ }^{41} \mathrm{Ca}$ this hypothesis could fully account for the missing intensity found by the experiment $[5,6]$. Surprisingly, in another case of $1 u$ REC - the decay of ${ }^{204} \mathrm{Tl}$ [8] - the opposite situation was observed. The intensity of the measured REC spectrum was found to be smaller by a factor of 4 than the predictions of the Zon and Rapoport model. Thus, no room for detour transitions was left, since their contribution is always positive in the model of Kalinowski.

To solve this conundrum, Pachucki et al. have undertaken a new approach to the description of the REC process [9]. The main ingredient of the proposed model was the description of the electromagnetic field in the length gauge [10] in contrast to the Coulomb gauge used in all previous calculations. The key point is that, although the predictions are gauge invariant, the particular length gauge is strongly preferred for the actual calculation in this case. One reason is a suppression of the nuclear contributions, which allows the detour transitions to be neglected. The new model was found to agree very well with experimental spectra of ${ }^{41} \mathrm{Ca}$ and ${ }^{204} \mathrm{Tl}$ [9].

In this paper, we report on REC measurements for the third case of $1 u$ transitions - the decay of ${ }^{81} \mathrm{Kr}$. This nucleus, with an intermediate mass and atomic number, located between ${ }^{41} \mathrm{Ca}$ and ${ }^{204} \mathrm{Tl}$, suits very well as a test case for the theory of radiative electron capture accompanying forbidden decays. In addition, this is the last case known where the radiation accompanying a first-forbidden unique $(1 u)$ decay can be measured for the pure ground-state to ground-state transition, which makes it experimentally feasible.

\section{A. Decay properties of ${ }^{81} \mathbf{K r}$}

The $7 / 2^{+}$ground state of ${ }^{81} \mathrm{Kr}$ decays with a probability of almost $100 \%$ to the $3 / 2^{-}$ground state of ${ }^{81} \mathrm{Br}$ with 
the half-life of $2.3 \times 10^{5} \mathrm{yr}$ [11]. This transition is of the first-forbidden unique type. The decay energy $Q_{\mathrm{EC}}$ amounts to $(280.8 \pm 0.5) \mathrm{keV}[11]$, so that the decay can proceed only by an electron capture process. A small branch of the ${ }^{81} \mathrm{Kr}$ decay feeds the first excited state of ${ }^{81} \mathrm{Br}$ at $276 \mathrm{keV}$ with the spin and parity of $5 / 2^{-}$. The branching for this first forbidden nonunique transition was measured by Axelsson et al. to be $3.0(2) \times 10^{-3}[12]$. However, the decay energy for this weak branch equals only $4.7 \mathrm{keV}$ and is much smaller than the $K$-electron binding energy in a bromine atom, which amounts to $B_{K}=13.47 \mathrm{keV}$. Therefore, the transitions to the excited state in ${ }^{81} \mathrm{Br}$ proceed only by electron captures from higher shells. In turn, the $K$-electron capture can occur only in a ground-state to ground-state transition. Thus, by a condition of coincidences with $\mathrm{Br} K \mathrm{x}$ rays one can select pure $1 u$ $K$-capture transitions between ground states of ${ }^{81} \mathrm{Kr}$ and ${ }^{81} \mathrm{Br}$. The maximum energy available for REC photons in the $K$ capture is given by $q_{K}=Q_{\mathrm{EC}}-B_{K}$, which leads to $q_{K}=267.3 \mathrm{keV}$.

\section{EXPERIMENTAL TECHNIQUE}

\section{A. ${ }^{81} \mathrm{Kr}$ source}

The activity of ${ }^{81} \mathrm{Kr}$ was collected at CERN-ISOLDE [13], using a proton beam of $1 \mathrm{GeV}$ energy that induced spallation reactions in a ${ }^{93} \mathrm{Nb}$ production target. The reaction products were ionized in a surface ion source, accelerated to a kinetic energy of $50 \mathrm{keV}$ and subsequently mass separated by means of the GPS separator tuned for the transmission of particles with $A=81$. The selected products were implanted in an aluminum catcher foil of $10 \mathrm{mg} / \mathrm{cm}^{2}$ thickness. Krypton ions, although produced in the reaction, are not ionized and extracted from the source with any significant efficiency. However, rubidium is readily ionized with high yield and it was thus primarily ${ }^{81} \mathrm{Rb}$ ions that were implanted, with some additional component of ${ }^{81} \mathrm{Sr}$. These implanted ions, having half-lives of $4.58 \mathrm{~h}$ and $22.2 \mathrm{~min}$, respectively, eventually decayed to ${ }^{81} \mathrm{Kr}$, forming the source of interest.

To avoid sputtering of already implanted atoms by the impinging beam, the position of the foil was changed. After the irradiation, the foil was left in the chamber for a few days to let the short-lived precursor activity decay out. The final source material was collected on two foils in two irradiation sessions, in 1998 and in 2000, yielding six active spots, each having the area of a few millimeters squared.

Two longer-lived contaminants were found in the source, stemming from incomplete suppression of neighboring isobars in the mass separator: ${ }^{82} \mathrm{Sr}\left(T_{1 / 2}=22.5\right.$ days $)$ and ${ }^{83} \mathrm{Rb}$ $\left(T_{1 / 2}=86.2\right.$ days $)$. Their $\gamma$-ray activity, in particular $\mathrm{Kr} K \mathrm{x}$ rays, makes the ${ }^{81} \mathrm{Kr}$ REC measurements very difficult. Instead of undertaking any chemical purification of the source, we decided to wait a few years until these contaminants have decayed. The activity of ${ }^{81} \mathrm{Kr}$ and of the contaminants was monitored. The $\gamma$ and $K x$-ray spectra of collected samples, measured in 2001 and 2003, are shown in Figs. 1 and 2.

Finally, in 2005 the influence of ${ }^{83} \mathrm{Rb}$ was found to be negligible. The active spots were carefully cut out from the catcher foils and placed on a Perspex disk $1 \mathrm{~mm}$ thick and
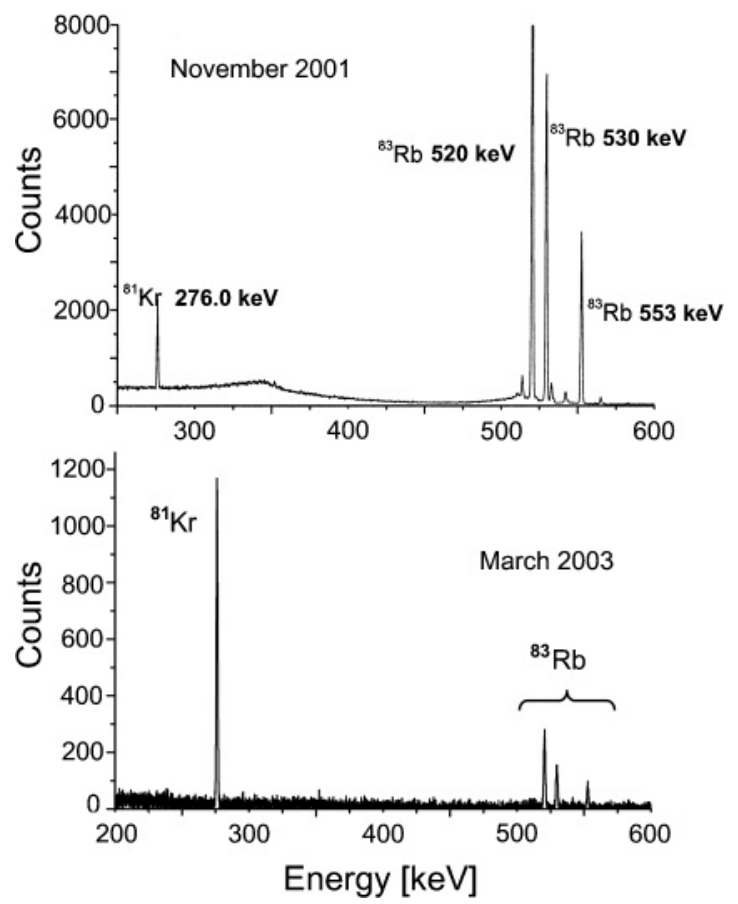

FIG. 1. Singles $\gamma$-ray spectra of the ${ }^{81} \mathrm{Kr}$ source taken in November 2001 (upper panel) and in March 2003 (lower panel). $\gamma$ transitions following decays of contaminant ${ }^{83} \mathrm{Rb}$ atoms are seen, as well as a $276 \mathrm{keV}$ line following a decay of ${ }^{81} \mathrm{Kr}$ to the $5 / 2^{-}$excited state in ${ }^{81} \mathrm{Br}$.

$2 \mathrm{~cm}$ in diameter and covered tightly with a thin mylar foil. The number of ${ }^{81} \mathrm{Kr}$ atoms in the final source was measured to be $4.1(2) \times 10^{15}$.

\section{B. Photon spectrometry}

Measurements were performed in the Institute of Experimental Physics at the Faculty of Physics of University of Warsaw. To select the $K$-capture component, the REC spectrum was measured in coincidence with the $\mathrm{Br} K \mathrm{x}$ rays. REC photons were recorded with an ORTEC GMX 45\% $\mathrm{Ge}$ detector. The $\mathrm{x}$ rays were measured by means of an ORTEC LOAX spectrometer. Both detectors had 0.5-mmthick beryllium windows.

The source was mounted between the two detectors in a close face-to-face geometry, with the surface covered with mylar foil directed toward the x-ray detector. The whole setup was placed inside a lead shielding of $10 \mathrm{~cm}$ thickness. The inside of the shielding was covered with 1.2-mm-thick cadmium sheets and 8-mm-thick copper plates.

A single DGF-4C CAMAC module [14] was used to process electronic signals from both detectors. The preamplifier outputs were directly connected to the two inputs of the DGF-4C module. Each input signal was digitized with $40 \mathrm{MHz}$ frequency followed by real-time digital signal processing which included time stamping. Output data were read out by a PC computer and stored on a hard disk. In the off-line analysis, the coincidence relationships between signals could be restored with the help of the time stamps. In addition, a $1 \mathrm{~Hz}$ pulser was connected to the test inputs of both preamplifiers 


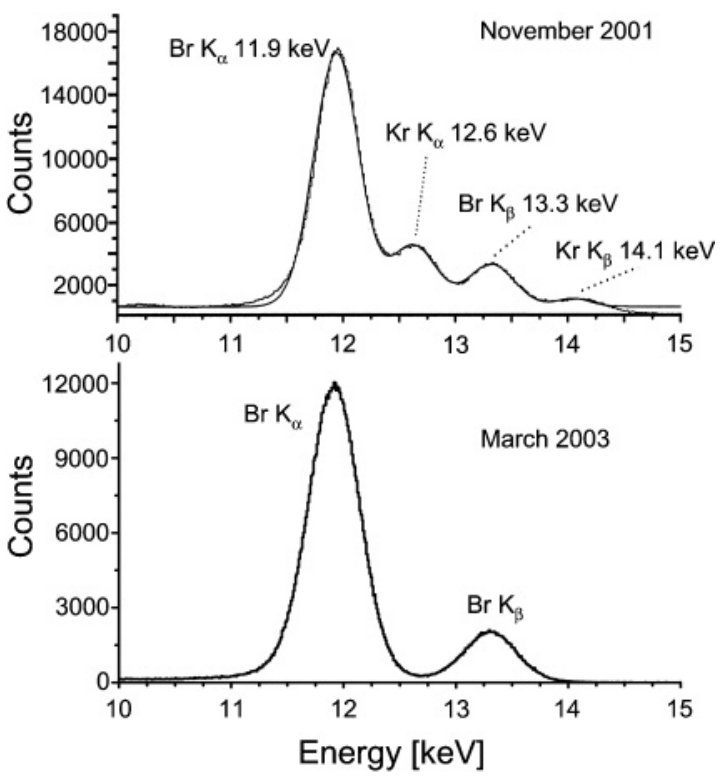

FIG. 2. Singles $K \mathrm{x}$-ray spectra of the ${ }^{81} \mathrm{Kr}$ source taken in November 2001 (upper panel) and in March 2003 (lower panel). In the upper spectrum contaminant $\mathrm{Kr} K \mathrm{x}$ rays originating from decays of ${ }^{83} \mathrm{Rb}$ can be seen.

and to a free running scaler. Comparison of the scaler readings with the number of counts recorded by the DGF-4C module allowed us to estimate and to monitor the dead time of the acquisition system.

The long coincidence sessions (1-2 days) were alternated with short (ca. $10 \mathrm{~min}$ ) singles measurements necessary for the $K \mathrm{x}$-ray intensity monitoring.

Two series of measurements were carried out. In the first one, in 2005, the total time of $\gamma-K \mathrm{x}$-ray coincidence runs amounted to $925.7 \mathrm{~h}$ ( $\simeq 38.6$ days). The average rate of singles $\mathrm{Br} K \mathrm{x}$ rays was determined to be 107.7(5) counts/s. After all runs, the ${ }^{81} \mathrm{Kr}$ source was removed and the background was measured in the same coincidence conditions for $113 \mathrm{~h}$.

In the second series of measurements, in 2007-2008, the $\gamma-K \mathrm{x}$-ray coincidences were collected for a total of 78 days. The average singles intensity of $\mathrm{Br} K \mathrm{x}$ rays over this period was found to be 95.1(5) counts/s. This time, however, for reasons explained in the following, the ${ }^{81} \mathrm{Kr}$ runs were alternated with background $\gamma-K x$-ray coincidences. The total time of background coincidence measurements was 71 days. The total time of the second experiment amounted to 190 days.

\section{Calibrations and corrections}

The calibrations of both Ge detectors, including the efficiency calibration of the GMX detector, were performed with help of standard sources: ${ }^{57} \mathrm{Co},{ }^{60} \mathrm{Co},{ }^{137} \mathrm{Cs},{ }^{152} \mathrm{Eu},{ }^{203} \mathrm{Hg}$, and ${ }^{241} \mathrm{Am}$. In order to avoid summing effects, the full-energy peak efficiency of the GMX detector was measured in a far geometry with all sources and in the close geometry, corresponding to the ${ }^{81} \mathrm{Kr}$ source position, only with ${ }^{241} \mathrm{Am},{ }^{203} \mathrm{Hg}$, and ${ }^{137} \mathrm{Cs}$, in which no summing occurs. Then, the far-geometry curve was scaled to the close-geometry position based on the efficiency ratios determined with the latter sources. The finally determined efficiency of the GMX detector at $100 \mathrm{keV}$ amounted to about $14 \%$ for both runs of ${ }^{81} \mathrm{Kr}$ measurements.

The performance of the coincidence circuitry was tested with a ${ }^{133} \mathrm{Ba}$ source measured in a far geometry to avoid summing effects. From the decay scheme of ${ }^{133} \mathrm{Ba}$ the probabilities of $\gamma$-line emission per $K$ capture were determined for the lines at $81,276,302,356$, and $384 \mathrm{keV}$. These values were compared with the measured $\gamma-K x$-ray coincidences, yielding a coincidence efficiency consistent with $100 \%$ within error bars of a few percent for all tested energies.

Since the REC spectrum of photons is continuous, it has to be corrected for the Compton scattering. To estimate the magnitude and shape of the corresponding correction, the response of the GMX detector to monoenergetic radiation had to be determined. First, the response was measured with the help of sources: ${ }^{241} \mathrm{Am},{ }^{57} \mathrm{Co},{ }^{203} \mathrm{Hg}$, and ${ }^{137} \mathrm{Cs}$. Then, the spectra were compared with Monte Carlo simulations performed with the GEANT package [15]. The effective dimensions of the detection setup were adjusted to obtain a satisfactory agreement between the measured and simulated spectra. This allowed calculation of the detector response to the radiation of arbitrary energy within the range of interest.

\section{Background}

Selection of the $K$ component of the REC spectrum requires coincidences with $K \mathrm{x}$ rays of the daughter atom. Usually, this condition practically removes any background contribution to the measured $\gamma$ spectrum. However, in the case of ${ }^{81} \mathrm{Kr}$, the energy values of $\mathrm{Br} K \mathrm{x}$ rays, 11.9 and $13.3 \mathrm{keV}$, unfortunately happen to overlap with $\mathrm{x}$-ray energies of heavy elements, like protactinium and thorium. Since some radioactive isotopes of these elements belong to natural radioactivity chains, traces of them appear in the surroundings, mainly in lead bricks. Coincidence conditions result in a selection of those $\gamma$ rays from their decays which are coincident with transitions strongly converted on the L-shell. An example of background $\gamma$ spectrum, measured without the ${ }^{81} \mathrm{Kr}$ source but with the same coincidence conditions, in particular with a gate on $\mathrm{Br} \mathrm{KX}$ energy range in the LOAX detector, is shown in Fig. 3.

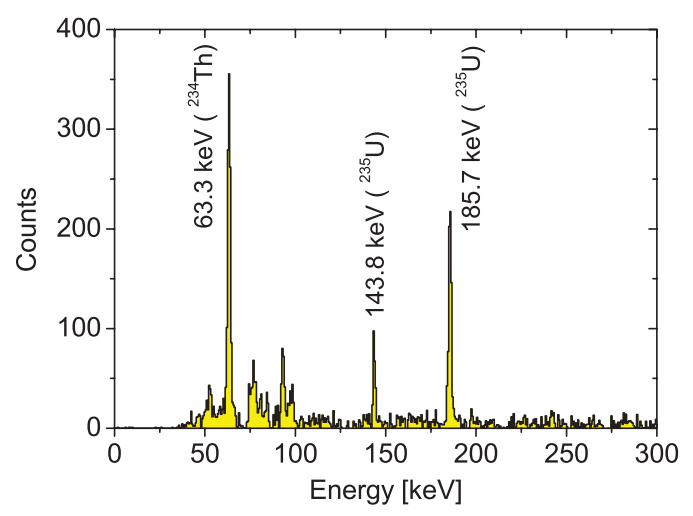

FIG. 3. (Color online) The background $\gamma$ spectrum gated by radiation in the $\mathrm{Br} K \mathrm{x}$-ray energy range detected in the LOAX detector. The total measurement time was 71 days. 
The strongest $\gamma$ lines present in Fig. 3 are identified as emitted by members of uranium-radium and actinium natural radioactivity chains, starting from ${ }^{238} \mathrm{U}$ and ${ }^{235} \mathrm{U}$, respectively. For example, a strong line at $63.3 \mathrm{keV}$ is emitted from ${ }^{234} \mathrm{~Pa}$ after the $\beta^{-}$decay of ${ }^{234} \mathrm{Th}$ (itself a decay product of ${ }^{238} \mathrm{U}$ ). This line is seen because of coincidence with an $E 2$ transition of $29.5 \mathrm{keV}$ which has a large $L$-shell conversion coefficient in protactinium. Another $L$-converted transition of $20.0 \mathrm{keV}$ $(M 1+E 2)$ in ${ }^{243} \mathrm{~Pa}$, is in coincidence with the $92.4 \mathrm{keV} \gamma$ line which can also be seen in the spectrum. Two other strong lines, at 143.8 and $185.7 \mathrm{keV}$, correspond to transitions between states in ${ }^{231} \mathrm{Th}$ fed by $\alpha$ decay of ${ }^{235} \mathrm{U}$. Both are coincident with a $19.6 \mathrm{keV} M 1+E 2$ transition which is strongly $L$ converted.

The contribution of natural background to the REC spectrum was identified after the first series of measurements when the coincidence run without the ${ }^{81} \mathrm{Kr}$ source was taken only for $113 \mathrm{~h}$. Therefore, in the second series, the coincident background runs were alternated with REC runs, so that a background spectrum with much higher statistics was accumulated.

\section{RESULTS}

\section{A. REC spectrum}

The $K$ component of the REC spectrum of ${ }^{81} \mathrm{Kr}$ was determined separately from both measurement series by applying the following procedure. First, the spectrum of time differences between $\gamma$-ray and $K \mathrm{x}$-ray events was created. The peak in this spectrum allows discrimination of the true $K \mathrm{x}-\gamma$ coincident events from a flat background representing random coincidences. Then, by appropriate gating on the $\mathrm{x}$-ray coordinate to select $\mathrm{Br} K \mathrm{x}$ rays and on the spectrum of time differences, the spectrum of $\gamma$ rays coincident with $\mathrm{Br} K \mathrm{x}$ rays was constructed for all runs measured with the ${ }^{81} \mathrm{Kr}$ source. This involved also gating on the $\mathrm{x}$-ray spectrum on the side of the $\mathrm{Br} K \mathrm{x}$-ray peak to account for possible coincident events which constitute a continuous spectrum underneath the $\mathrm{Br} \mathrm{x}$-ray lines. Exactly the same gating procedure was used to determine the $\gamma$ spectrum of background coincidences, discussed in the previous section. After normalization, the background spectrum was subtracted from the former one, yielding the raw REC spectrum of ${ }^{81} \mathrm{Kr}$ accompanying the $K$-shell electron capture. As an example, the spectra from the second experiment are shown in Fig. 4. The total number of counts in the REC spectrum is 16800 , which corresponds to about nine coincident events per hour.

In the next step, the correction for Compton scattering is introduced such that the spectrum is presented in fullenergy-peak efficiencies. The calculated contributions of each $\gamma$-energy bin are subtracted one by one, starting from the highenergy end (a peeling-off method). This correction is largest at low energy where contributions from all higher-energy bins add up and amount to about $30 \%$. The corrected REC spectrum is shown in Fig. 4 by the points with error bars.

Finally, the absolute normalization of the REC spectrum is made. Each bin of the Compton-corrected REC spectrum is divided by the corresponding full-energy-peak efficiency of the GMX detector and by the total number of $\mathrm{Br} K \mathrm{x}$ rays recorded by the LOAX detector during all ${ }^{81} \mathrm{Kr}$ coincidence runs. This number was determined from the singles $K \mathrm{x}$-ray

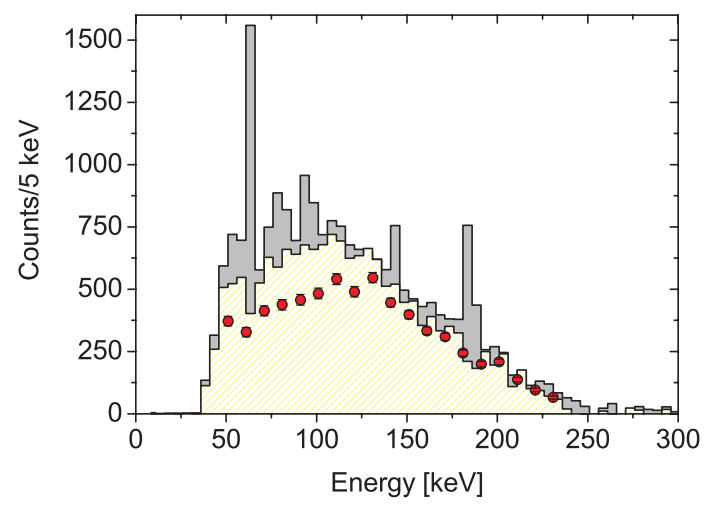

FIG. 4. (Color online) $\gamma$ energy spectrum coincident with $\mathrm{Br} K \mathrm{x}$ rays from the second series of measurements. The upper histogram was extracted from runs with the ${ }^{81} \mathrm{Kr}$ source. The contribution of background lines can be seen. The lower histogram shows the result after background subtraction. The circles filled in red illustrate the result of Compton correction and represent the measured $K$ component of the ${ }^{81} \mathrm{Kr}$ REC spectrum.

measurements and includes corrections for the dead time in both the singles and coincidence runs. Such a procedure yields the probability distribution of the REC photon energy normalized to ordinary, nonradiative $K$-capture rate. This final REC energy spectrum can be directly compared to theoretical predictions. The resulting distributions from two series of measurements are presented in Fig. 5.

\section{B. Theoretical models}

In a very general way, the probability of radiative electron capture from the $1 S$ state ( $K$ capture) in which a photon in the energy range $(k, k+d k)$ is emitted, per ordinary, nonradiative $K$ capture can be written as [1]

$$
\frac{d w_{K}^{\mathrm{REC}}(k)}{w_{K}}=\frac{\alpha}{\pi\left(m_{e} c^{2}\right)^{2}} \frac{k\left(q_{K}-k\right)^{2}}{q_{K}^{2}} R_{K}(k) d k,
$$

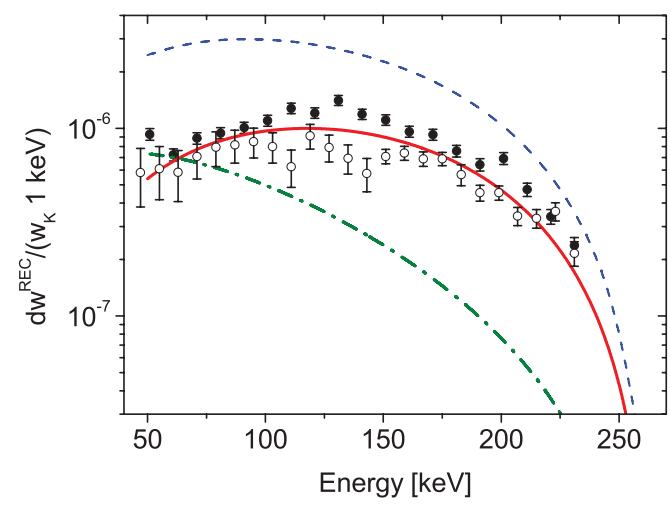

FIG. 5. (Color online) The intensity of the $K$ component of the REC spectrum of ${ }^{81} \mathrm{Kr}$ per ordinary, nonradiative $K$ capture and per $1 \mathrm{keV}$. Open and closed circles represent the first and the second series of measurements, respectively. The solid line shows the prediction of the model by Pachucki et al. [9], while its Coulomb-free limit is represented by the dashed line. The dash-dotted line illustrates the prediction of the Zon and Rapoport model $[3,4]$. 
where $\alpha$ is the fine structure constant, $m_{e} c^{2}$ is the electron rest energy, $q_{K}$ is the photon end-point energy, and the dimensionless function $R_{K}(k)$ is the shape factor of the spectrum. In the simplest Coulomb-free, nonrelativistic approximation, valid for allowed nuclear transitions, $R_{K}(k) \equiv 1$ [16].

In a more appropriate theoretical approach which takes into account both relativistic effects and the influence of the Coulomb field, and which is valid for first-forbidden unique nuclear transitions, the shape factor is given by

$$
R_{K}^{1 u}(k)=\left(1-\frac{k}{q_{K}}\right)^{2} R_{K}^{(1)}(k)+\left(\frac{k}{q_{K}}\right)^{2} R_{K}^{(2)}(k),
$$

where the functions $R_{K}^{(1)}$ and $R_{K}^{(2)}$ describe the effects of the Coulomb interaction between the nucleus and the radiating electron. Usually, these functions have to be calculated numerically for each specific case.

The first advanced description of REC in forbidden transitions was provided by Zon and Rapoport [3,4], who extended a framework for allowed decays, developed previously by Glauber and Martin [17,18], and generalized it to nuclear transitions of any order of forbiddenness. In this model both functions, $R_{K}^{(1)}$ and $R_{K}^{(2)}$, approach unity in the Coulombfree limit $(Z \rightarrow 0)$. The model of Zon and Rapoport was found to fully reproduce the earlier results of Glauber and Martin for allowed decays, which were quite well confirmed experimentally [1]. However, both the full and the Coulombfree versions of this model failed to describe the REC spectrum measured in the case of $1 u$ decays in ${ }^{41} \mathrm{Ca}$ and ${ }^{204} \mathrm{Tl}[2,8]$.

This observation motivated Pachucki et al. to reconsider the problem of REC in forbidden decays following a different approach [9]. The important conclusion from that work was that, although final results must not depend on a particular choice of gauge for the electromagnetic field, the Coulomb gauge applied by previous authors has rather unfortunate consequences when, as exemplified in the work of Zon and Rapoport, the approximations may later result in diverging terms. In addition, in the Coulomb gauge one has to include contributions from nuclear degrees of freedom. It turned out that the adoption of a different gauge for the electromagnetic field, the so-called length gauge, simplifies calculations considerably and avoids, in fact, the difficulties mentioned before. In particular, it was demonstrated that in the length gauge the contribution from the nuclear (detour) transitions can be neglected [9].

The results of Pachucki et al. confirmed all previous predictions for allowed transitions as well as the detailed form of the function $R_{K}^{(1)}$, appearing in Eq. (2). However, a different form was derived for the second function- $R_{K}^{(2)}$, which is the one affected by a diverging term in the model of Zon and Rapoport. In particular, the Coulomb-free limit for this function was found to be [9]

$$
\lim _{Z \rightarrow 0} R_{K}^{(2)}(k)=1+\frac{m_{e} c^{2}}{k}+2\left(\frac{m_{e} c^{2}}{k}\right)^{2},
$$

which obviously differs from 1 .

The model of Pachucki et al. was found to be in almost perfect agreement with measured spectra in ${ }^{41} \mathrm{Ca}$ and in ${ }^{204} \mathrm{Tl}$

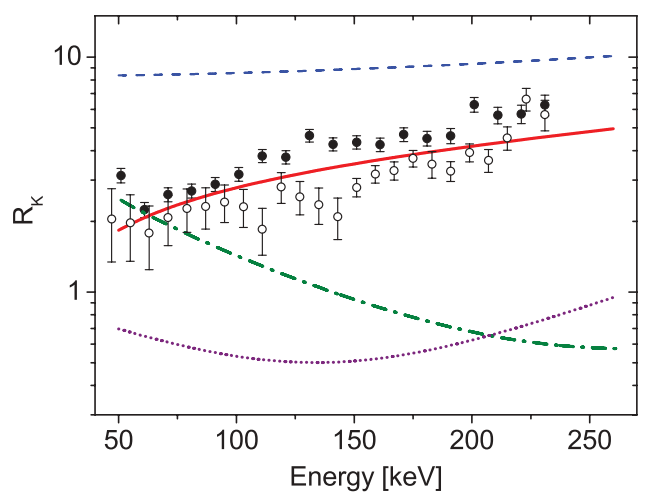

FIG. 6. (Color online) The shape factor for the $K$ component of the first-forbidden unique REC in ${ }^{81} \mathrm{Kr}$. Open and closed circles represent the first and the second series of measurements, respectively. The meaning of the theoretical lines is the same as in Fig. 5, while the additional dotted line shows the Coulomb-free version of the Zon and Rapoport model.

[9]. Its prediction for the present case of ${ }^{81} \mathrm{Kr}$ is plotted in Fig. 5 with the solid line. The results of the Coulomb-free limit and of the Zon and Rapoport model are also shown.

\section{1S shape factor}

The shape of the REC spectrum, contributions of different terms, and comparison with data can be shown in detail by plotting the dimensionless shape factor of the spectrum, $R_{K}(k)$. The experimental shape factors were extracted from the determined REC spectra with the help of Eq. (1). The values obtained are shown in Fig. 6, which contains also the theoretical predictions.

As seen in Figs. 5 and 6, there is a systematic difference between the results obtained from the two experiments. The collected statistics in the first series was smaller, which is partly reflected in larger errors. In addition, the correction for background was less accurate in the first experiment, which could result in a systematic shift. Since the investigated effect is small and the rate of coincidences was very low, a proper control of possible systematic effects was difficult. We note, however, that each spectrum taken separately would lead to the same conclusion. The prediction of Pachucki et al. reproduces quite well both experimental spectra, in contrast to the model by Zon and Rapoport.

\section{Total intensity}

The total probability of the $K$ component of the REC spectrum per nonradiative $K$ capture, calculated by integrating the spectrum from $50 \mathrm{keV}$ to the end point, is given in Table I together with values obtained from theoretical models. The average of the total intensity from two measurements equals $1.42(22) \times 10^{-4}$ per $K$ capture.

The values of total intensity from both measurements, as well as their average, are closest to the prediction of Pachucki et al. It is interesting to note the large difference between the Coulomb-free limits of the two theories. In the case of 
TABLE I. The total probability for the $K$ component of REC per $K$ capture in ${ }^{81} \mathrm{Kr}$, integrated from $50 \mathrm{keV}$ to the spectrum end point, in units of $10^{-4}$, obtained from the first (a) and the second (b) experiment. The results of a full calculation, as well as the Coulombfree version $(\mathrm{CF})$, are given for both theoretical models.

\begin{tabular}{llllll}
\hline \hline \multirow{2}{*}{ Experiment } & \multicolumn{2}{c}{ Pachucki et al. } & & \multicolumn{2}{c}{ Zon and Rapoport } \\
\cline { 2 - 3 } & Full & CF & & Full & CF \\
\hline (a) $1.2(1)$ & 1.37 & 4.00 & & 0.60 & 0.26 \\
(b) $1.64(8)$ & & & & \\
\hline \hline
\end{tabular}

Pachucki et al., this approximation overestimates the spectrum intensity. The inclusion of Coulomb and relativistic effects reduces the intensity to a value that almost perfectly agrees with the experiment. The model of Zon and Rapoport behaves in the opposite way. The Coulomb-free limit lies below the full calculation, which in turn is still much lower than the experimental values.

\section{CONCLUSIONS}

We have measured the spectrum of photons accompanying the first-forbidden unique electron capture decay of ${ }^{81} \mathrm{Kr}$. By detecting $\gamma$ rays in coincidence with $\mathrm{Br} K \mathrm{x}$ rays we have selected the pure $K$ component of radiative electron capture taking place between the ground states of ${ }^{81} \mathrm{Kr}$ and ${ }^{81} \mathrm{Br}$. From the measured spectra we have extracted the absolute probability of REC emission per normal, nonradiative $K$-electron capture as a function of photon energy, as well as the shape factor of the photon spectrum. Both the shape and intensity of the REC spectrum are found to be well reproduced by the recent theoretical model of Pachucki et al. [9], while strong disagreement is found with predictions of the old model by Zon and Rapoport $[3,4]$. Thus, ${ }^{81} \mathrm{Kr}$ is the third case displaying $\mathrm{REC}$ in a $1 u$ nuclear transition, in addition to ${ }^{41} \mathrm{Ca}$ and ${ }^{204} \mathrm{Tl}$, which strongly supports the approach taken by Pachucki et al. In contrast, the model of Zon and Rapoport fails to describe correctly the probability and shape of the REC spectrum in all these three cases.

The important feature of the first-forbidden unique decays is that they are governed predominantly by a single nuclear matrix element. In the probability ratio of the radiative capture to the ordinary, nonradiative one, which is determined in experiment, this nuclear matrix element cancels out. Thus, the result should not depend on detailed knowledge of nuclear wave functions and should be fully determined by the electroweak sector alone. An important consequence is that the theoretical models discussed do not contain any adjustable parameters. The predictions are fully determined by the mass and atomic numbers of the decaying nucleus and by the maximal photon energy, related to the decay energy $Q_{\mathrm{EC}}$.

The main difference between the two theoretical models discussed is the selected gauge of electromagnetic field. While Zon and Rapoport use the Coulomb gauge, Pachucki et al. apply the length gauge. Our conclusion is that the latter is preferred. The reason is that calculations in the length gauge are technically simpler and do not require additional approximations. Also, Zon and Rapoport introduced approximations that were not valid and led to a diverging term which was found to be responsible for the final failure in comparison with the experiment. Moreover, in the length gauge the contribution from nuclear degrees of freedom to the emitted radiation can be shown to be negligible, while this is not true in the case of the Coulomb gauge.

The $1 u$ decays of ${ }^{41} \mathrm{Ca},{ }^{81} \mathrm{Kr}$, and ${ }^{204} \mathrm{Tl}$ are the only ones known where the pure transition between ground states can be selected. Thus, only in these cases can the weak radiative branch be determined with sufficient accuracy. Additional tests of this alternative description of REC are in principle possible with transitions of second-forbidden nonunique type ( $2 n u: \Delta J=2, \pi_{i} \pi_{f}=+1$ ). To this class belong ${ }^{59} \mathrm{Ni}$ and ${ }^{137} \mathrm{La}$ in which the $K$ component of the REC spectrum was measured $[19,20]$. The results obtained for ${ }^{59} \mathrm{Ni}$ disagree with the Zon and Rapoport predictions, while the spectrum for ${ }^{137} \mathrm{La}$ could not be shown to contradict them. However, in the case of $2 n u$ decays, the REC spectrum depends on one additional parameter that represents a ratio of nuclear matrix elements. Since these matrix elements are not known and are difficult to estimate, this parameter adds an extra degree of freedom. In consequence, the comparison between an experiment and the theory is not as unambiguous as in the case of $1 u$ transitions. Nevertheless, in the future we plan to compare the measured spectra of ${ }^{59} \mathrm{Ni}$ and ${ }^{137} \mathrm{La}$ with predictions of the new model of Pachucki et al.

\section{ACKNOWLEDGMENTS}

We are grateful to the CERN-ISOLDE staff for assistance and patience during irradiations to produce the samples of ${ }^{81} \mathrm{Kr}$.
[1] W. Bambynek, H. Behrens, M. H. Chen, B. Crasemann, M. L. Fitzpatrick, K. W. D. Ledingham, H. Genz, M. Mutterer, and R. L. Intemann, Rev. Mod. Phys. 49, 77 (1977).

[2] P. Hornshøj, T. Batsch, Z. Janas, M. Pfützner, A. Płochocki, and K. Rykaczewski, Nucl. Phys. A 472, 139 (1987).

[3] B. A. Zon and L. P. Rapoport, Yad. Fiz. 7, 528 (1968) [Sov. J. Nucl. Phys. 7, 330 (1968)].

[4] B. A. Zon, Yad. Fiz. 13, 963 (1971) [Sov. J. Nucl. Phys. 13, 554 (1971)].

[5] Ł. Kalinowski, Z. Janas, M. Pfützner, A. Płochocki, P. Hornshøj, and H. L. Nielsen, Nucl. Phys. A 537, 1 (1992).
[6] Ł. Kalinowski, Nucl. Phys. A 551, 473 (1993).

[7] G. W. Ford and C. F. Martin, Nucl. Phys. A 134, 457 (1969).

[8] J. Kurcewicz, M. Pfützner, P. Hornshøj, H. L. Nielsen, and B. Szweryn, Nucl. Phys. A 728, 3 (2003), and references therein.

[9] K. Pachucki, U. D. Jentschura, and M. Pfützner, Phys. Rev. C 75, 055502 (2007).

[10] W. R. Johnson, Atomic Structure Theory, Lectures on Atomic Physics (Springer, Berlin, 2007).

[11] C. M. Baglin, Nucl. Data Sheets 109, 2257 (2008).

[12] H. Axelsson et al., Phys. Lett. B 210, 249 (1988).

[13] E. Kugler, Hyperfine Interact. 129, 23 (2000). 
[14] B. Hubbard-Nelson, M. Momayezi, and W. K. Warburton, Nucl. Instrum. Methods Phys. Res. A 422, 411 (1999).

[15] J. Allison et al., IEEE Trans. Nucl. Sci. 53, 270 (2006); [http://geant4.cern.ch].

[16] P. Morrison and L. Schiff, Phys. Rev. 58, 24 (1940).
[17] R. J. Glauber and P. C. Martin, Phys. Rev. 104, 158 (1956).

[18] P. C. Martin and R. J. Glauber, Phys. Rev. 109, 1307 (1958).

[19] Z. Janas et al., Nucl. Phys. A 524, 391 (1991).

[20] M. Pfützner et al., Nucl. Phys. A 611, 270 (1996). 